\title{
DESPESAS DOS ESTADOS E DO DISTRITO FEDERAL COM OS SERVIÇOS DE EDUCAÇÃO, EM 1943
}

No intuito de estudar as despesas com a educação, em todo o país, vem o Instituto Nacional de Estudos Pedagógicos procedendo, cada ano, a análise dos orçamentos do Distrito Federal e dos Estados, na parte referente aos serviços de ensino e de difusão cultural, por eles respectivamente mantidos.

Por esse trabalho, pode-se facilmente verificar, não só o montante dos dinheiros públicos destinados à educação, pelas unidades federadas, como também a sua aplicação pelos vários ramos de ensino e de educação extraescolar.

Por ser muito variável a organização dos serviços, em cada Estado, e variável também a compreensão de algumas das dotações, a que se ligam despesas de aparelhamento - como as destinadas, por exemplo. à construção e à conservação de prédios públicos - é de compreender-se que, nesses casos, o I.N.E.P. devia proceder por estimativa. Assim também, em relação às despesas com a administração, visto que, na maioria dos Estados, os serviços de educação pertencem, com outros, a uma e mesma secretaria ou departamento de administração.

Ainda assim, o estudo constitui tentativa muito útil, para visão geral do movimento das dotações; e, pelo cotejo dos vários índices, em exercícios sucessivos, para a percepção das tendências que os sistemas regionais de educação vêm apresentando.

\section{AS DESPESAS COM O SERVIÇO DE EDUCAÇÃO NO PERÍODO DE 1939-1943}

Antes de indicar os resultados do estudo dos orçamentos relativos ao exercício de 1943, é de notar o rápido crescimento geral das dotações, destinadas aos serviços de educação, pelos Estados e Distrito Federal, nos últimos anos. 
Em 1939, somavam elas 398 milhões de cruzeiros; já no ano seguinte, alcançavam 459 milhões; no ano de 1941, elevavam-se a 493 milhões. Em 1942 não apresentavam, porém, tão forte aumento relativo, pois registraram 502 milhões e 962 mil cruzeiros. No ano de 1943, voltaram a crescer acentuadamente, atingindo ao montante de 563 milhões.

O aumento relativo neste período de cinco anos foi de cerca de $40 \%$, $o$ que dá o acréscimo médio anual de $8 \%$. De 1942 para 1943, o aumento foi de quase $12 \%$.

Duas condições de ordem geral têm condicionado o aumento das dotações, em cada unidade federada: o aumento progressivo dos orçamentos de receita, como seria natural; e, ainda, a maior ou menor atenção dada pela administração respectiva aos problemas da educação. Estas condições ressaltam quando se comparam os números, em mais alongado período, como o de 1932 a 1943.

\section{O ACRÉSCIMO DAS DESPESAS NO PERÍODO DE 1932 A 1943}

No ano de 1932, as despesas das várias unidades federadas com os serviços de educação, montaram a 222 milhões; em 1940, haviam dobrado, atingindo os orçamentos a 459 milhões. No ano passado, as dotações subiram já a 563 milhões.

Os orçamentos cresceram, assim, de $100 \%$, no período de 1932 a 1940, ou seja, em nove anos; nos últimos cinco anos, aumentaram, porém de $40 \%$, não tanto pelo crescimento proporcional dos serviços, como especialmente pelo reajustamento de vencimentos do pessoal docente, criação de novos serviços e melhoria dos já existentes. O aumento de matrícula, no ensino em geral, no mesmo período de nove anos não foi maior que $64 \%$, para aumento de despesa igual a $100 \%$. Nos últimos cinco anos, o acréscimo de matrícula foi de pouco mais de $15 \%$ para aumento de, como vimos, $40 \%$ de despesa.

Mas é certo também que houve maior desenvolvimento dos ramos de ensino do $2 .^{\circ}$ grau, por sua natureza e organização de mais oneroso custeio.

Não se apresenta, pois, pelas razões apontadas, relação absoluta entre o movimento de despesa e o de crescimento de matrícula. Essa relação aparece, porém, mais claramente, quando se examinam alguns índices, ainda de ordem geral, referentes a maior eficiência do trabalho escolar, como sejam os de freqüência e conclusões de curso. 
Quanto a estas, duplicaram, para todo o país, quando duplicada a despesa; e mantiveram sensivelmente a mesma proporção do aumento por esta última apresentada nos últimos anos.

O estudo particularizado para cada um dos Estados nos revela que, para muitos deles, o ritmo de acréscimo da própria matrícula manteve proporção com os das despesas da educação. Assim, para o Amazonas, Maranhão, Paraíba, Piauí e Rio Grande do Norte, que aumen-taram a matrícula de suas escolas no dobro, ou em mais do dobro, no período de 1932-1939; pròximamente, também, para Santa Catarina, Paraná, Pará, Mato Grosso e Sergipe.

O aumento das despesas dos Estados e do Distrito Federal, com os serviços da educação, do ano de 1932 para o de 1943, foi o seguinte:

\begin{tabular}{|c|c|c|c|}
\hline . & $\begin{array}{c}\text { Em } 1932 \\
\text { Cr\$ }\end{array}$ & $\begin{array}{c}\text { EM } 1943 \\
\text { Cr\$ }\end{array}$ & $\begin{array}{c}\text { AUMENTO } \\
\text { RELATIVO (\%) }\end{array}$ \\
\hline Distrito Federal........... & 33200978,00 & 116577454,00 & 251 \\
\hline 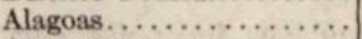 & 2130300,00 & 4276753,00 & 101 \\
\hline 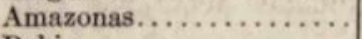 & 1720058,00 & $4982 \quad 189,00$ & 190 \\
\hline 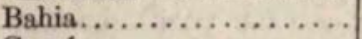 & $11^{\dagger} 351837,00$ & 23459131,00 & 107 \\
\hline 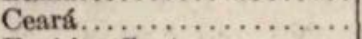 & $2,772892,00$ & 8024880,00 & 189 \\
\hline Espírito Santo........... & 3798598,00 & 7409630,00 & 95 \\
\hline 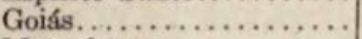 & 1383712,00 & 3630620,00 & 162 \\
\hline Maranhão............... & 1192174,00 & 3407300,00 & 186 \\
\hline Mato Grosso ................ & 1636550,00 & 3790520,00 & 132 \\
\hline Minas Gerais............ & 33203223,00 & 51790737,00 & 56 \\
\hline 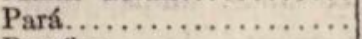 & 4385134,00 & 10993850,00 & 151 \\
\hline Paraíba.................. & $3024 \cdot 546,00$ & 6276048,00 & 108 \\
\hline 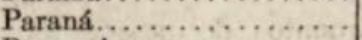 & 5706846,00 & 18234923,00 & 220 \\
\hline Pernambuco .............. & 7309554,00 & $12 \quad 272 \quad 370,00$ & 68 \\
\hline Piauí.................. & 1130539,00 & 4389366,00 & 288 \\
\hline Rio de Janeiro........... & $8359,912,00$ & 24427630,00 & 192 \\
\hline Rio Grande do Norte.... & $1754 \quad 190,00$ & 4295003,00 & 145 \\
\hline Rio Grande do Sul....... & 12342752,00 & $43 \quad 179284,00$ & 250 \\
\hline Santa Catarina............. & 2811083,00 & 12569289,00 & 347 \\
\hline São Paulo................ & 81241999,00 & 194855554,00 & 140 \\
\hline Sergipe................ & 2005930,00 & 4189556,00 & 109 \\
\hline Brasri............ & 222462807.00 & 563032087,00 & 153 \\
\hline
\end{tabular}

QUOTA DAS DESPESAS GERAIS COM A EDUCAÇÃO

O confronto do total das dotações para os serviços da educação, em cada Estado e no Distrito Federal, com os respectivos totais dos orçamentos de receita, apresenta-se, por outro lado, muito ilustrativo. 
Deve-se notar que esse confronto deixa de ter maior valor, se fôr feito com a receita total, sem maior indagação quanto à inclusão, nela, da renda de serviços industriais, sempre muito variável de uma para outra unidade federada. Os casos dos Estados do Rio Grande do Sul, São Paulo e Minas Gerais são típicos a este respeito. Excluída a renda dos serviços industriais, a receita de Minas desce de 406 milhões para 311: e do Rio Grande do Sul, de 451 milhões para 269; a de São Paulo, de um bilhão e 287 milhões, para 994 milhões.

Em outros Estados, ao contrário, ela entra em pequena proporção. E, como essa renda é consumida na manutenção dos próprios serviços industriais de onde provém, é claro que não deverá ser levada em consideração, no estudo comparativo das despesas das várias unidades.

Em relação à receita global, as despesas orçadas para a educação nos Estudos e Distrito Federal, no ano de 1943, representaram 15,86\%, contra $15,36 \%$, no ano anterior e $15,07 \%$ no ano de 1941 . Excluídas as rendas industriais, a mesma taxa se eleva, porém, para $19,47 \%$, contra $18,71 \%$, no ano anterior, e 18,02\% em 1941.

Tomada, porém, a "renda tributária de impostos e taxas", critério mais seguro para estudo comparativo entre as várias unidades federadas, a percentagem de despesas com os serviços gerais de educação, sobe de muito. Ela foi, no exercício de 1943 , de $23,83 \%$, que significa que, no conjunto, quase uma quarta parte dos tributos recolhidos estão sendo empregados em serviços de educação.

Mas a percentual referida é taxa média, e, assim de pequeno valor prático. A referida cota varia muito de uma para outra unidade. Chega a ser de $38,59 \%$, no Estado do Pará; mantém-se em 29,03\% no Distrito Federal; 27,61\%, no Paraná; e 27,43\% em Santa Catarina. Permanece ainda, em 25,88\% no Sergipe; 25,11 \% no Espírito Santo, e 24,03\%, na Paraíba. Mas desce para 18,86, em Minas Gerais; 17,55, em Pernambuco; 14,90, no Maranhão e 14,47 em Goiás. É o que se poderá ver, discriminadamente, nas tabelas que são apresentadas adiante.

A "renda tributária de impostos e taxas" se desdobra, como é óbvio, na de "impostos" e na de "taxas". E a consideração particula-rizada da primeira também importa, porquanto nela é que se baseiam as cotas estabelecidas pelo Convênio Nacional de Ensino Primário.

Por esse Convênio, celebrado entre a União, os Estados e o Distrito Federal, deverão estes gastar, só com o ensino primário, a partir do 
exercício atual de 1944 , cota igual a $15 \%$ da respectiva receita tributária de impostos; e essa cota se elevará, ano a ano, até que se mantenha em $20 \%$, no mínimo, resultado que deverá ser atingido em 1949.

Ainda em 1943, o total de gastos com o ensino primário, representava apenas, $14,26 \%$, do montante geral da renda de impostos recolhida pelos Estados e Distrito Federal. Ainda aí, a variação percentual de uma para outra unidade federada era muito grande. Oscilava entre $24,71 \%$, no Pará, e 7,45 \%, em Goiás. Ultrapassavam $20 \%$ apenas os Estados do Pará, Santa Catarina e Paraná. Ultrapassavam $15 \%$, além das unidades já mencionadas, o Distrito Federal, com 17,70\%; Mato Grosso, com 17,60\%; Espírito Santo, com 17,15\%; Paraíba, com 17,07 \%, e Rio de Janeiro, com 16,03\%.

\section{DISTRIBUIÇÃO GERAL DAS DESPESAS COM OS SERVIÇOS DE EDUCAÇÃO}

As dotações consignadas nos orçamentos de 1943 ,para os serviços gerais de educação, e que, montavam como vimos, a 563 milhões de cruzeiros, apresentavam a seguinte distribuição geral:

\begin{tabular}{|c|c|c|}
\hline & $\mathrm{Cr} \$$ & $\%$ \\
\hline Administração escolar ................................... & 47.041 .328 & 8,36 \\
\hline 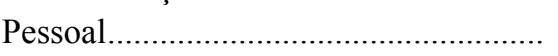 & 397.470 .023 & 70,59 \\
\hline Prédios e aparelhamentos........................... & 26.055 .967 & 4,63 \\
\hline Material de consumo.................................. & 24.665 .494 & 4,38 \\
\hline Assistência ao escolar ............................ & 14.662 .780 & 2,60 \\
\hline 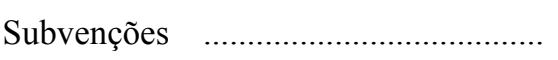 & 12.603 .740 & 2,24 \\
\hline 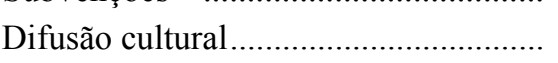 & 19.371 .687 & 3,44 \\
\hline 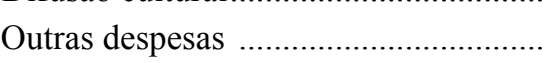 & 21.161 .068 & 3,76 \\
\hline $\begin{array}{ll}\text { Total ........ } \\
563.032 .087 & 100,00\end{array}$ & & \\
\hline
\end{tabular}

É de notar que é muito grande a variação das cotas percentuais, em cada uma dessas rubricas gerais, de uma para outra unidade federada.

Para a administração, consignou o Distrito Federal, 21,63 \% de suas dotações gerais para a educação; a Bahia, 10,70 \%; Mato Grosso, 9,66 \%, e Espírito Santo, 9,20 \%; ao passo que São Paulo gastou 4,73\%; Minas Gerais, 4,51\%, e Rio Grande do Sul, 4,08\%. 
As dotações com o pessoal de ensino representaram 57,85 \% no Distrito Federal; 64,06\% no Amazonas; e 66,91 em Sergipe. Em todas as demais unidades federadas representavam importe maior que $70 \%$, sendo que, no Ceará, era igual a $81,62 \%$.

Muito variáveis foram igualmente as cotas para material de consumo, assistência ao escolar, difusão cultural e subvenções, como poderá ser verificado nas tabelas que acompanham estes comentários.

DISTRIBUIÇÃO PELOS VÁRIOS RAMOS E GRAUS DF. ENSINO

Com o ensino propriamente dito, ou seja excluídas as despesas com os serviços de difusão cultural, as dotações dos Estados e do Distrito Federal, no ano de 1943, foram de 492.712 .526 cruzeiros.

O ensino primário, como seria natural, absorveu a maior cota, com 309 milhões; seguiu-se-lhe o ensino superior, com 40 milhões; o ensino normal, com 29 milhões; e o ensino secundário com mais de 25 milhões.

Assim distribuíram os orçamentos de 1943, nos Estados e Distrito Federal, suas dotações para o ensino, propriamente dito:

\begin{tabular}{|c|c|c|}
\hline Ensino & $\mathrm{Cr} \$$ & $\%$ \\
\hline Primário $. . . \ldots \ldots \ldots \ldots \ldots \ldots . . . . . .$. & $309.008 .886,00$ & 62,72 \\
\hline Secundário $\ldots . . \ldots \ldots \ldots \ldots$ & $25.433 .599,00$ & 5,16 \\
\hline Superior $\ldots \ldots \ldots \ldots \ldots \ldots \ldots$ & $40.065 .338,00$ & 8,13 \\
\hline Normal...$\ldots \ldots \ldots \ldots \ldots \ldots$ & $29.122 .834,00$ & 5,91 \\
\hline Tec. Industrial ............... & $21.625 .323,00$ & 4,39 \\
\hline Agrícola $\ldots \ldots \ldots \ldots \ldots \ldots \ldots$ & $23.843 .593,00$ & 4,84 \\
\hline Emendativo ................ & $15.828 .024,00$ & 3,21 \\
\hline Adultos...$\ldots \ldots \ldots \ldots \ldots \ldots$ & $1.883 .890,00$ & 0,38 \\
\hline Comercial .................. & $690.040,00$ & 0,14 \\
\hline Diversos $\ldots \ldots \ldots \ldots \ldots \ldots \ldots$ & $25.210 .999,00$ & 5,12 \\
\hline Total $\ldots \ldots \ldots \ldots \ldots \ldots$ & $492.712 .526,00$ & 100,00 \\
\hline
\end{tabular}

Como poderá ser visto nas tabelas a seguir, não foi possível discriminar o quantum dos gastos com o ensino secundário no Distrito Federal e no Estado do Rio de Janeiro, porque as dotações são aí globais para esse grau de ensino e o ensino normal; não apresentavam dotações para o ensino superior, industrial, agrícola, comercial e supletivo de adultos, várias unidades. 
DESPESA "PER CAPITA" COM OS SERVIÇOS DE EDUCAÇÃO

Pelo estudo geral dos orçamentos em apreço, verifica-se que a média de despesa "per capita", em relação aos serviços públicos, dessas unidades, em todo o país, teria sido, em 1943, de Cr\$ 82,45 anuais. Como todas as demais médias, essa representa apenas indicação de ordem muito geral. São enormes as variações regionais, pois oscilam de Cr\$279,54, no Distrito Federal, e Cr\$ 170,99 no Estado de São Paulo, a Cr\$ 20,88, no Maranhão e Cr\$ 18,84, no Ceará.

O mesmo se dirá para a despesa "per capita" com os serviços de educação. Em média, para todo o país, foi ela, em 1943, de Cr\$13,02. A variação, porém, foi ainda em maior proporção, pois oscilou de Cr\$62,89, no Distrito Federal, a Cr\$ 2,64 no Estado do Maranhão.

É de notar que estes dados se referem apenas às despesas constantes dos orçamentos dos Estados e do Distrito Federal. Incluídas as despesas da União, e as dos municípios, para o mesmo fim, a média dos gastos "per capita", no país, subirá a cerca de Cr\$18,00.

Não se apuraram ainda, de modo completo, para o exercício de 1943, as despesas da União e dos municípios com os serviços de educação.

Nas páginas a seguir, apresentam-se vários dos quados que resumem o estudo organizado pelo Instituto Nacional de Estudos Pedagógicas, em sua Seção de Inquéritos e Pesquisas. 
QUADRO I

RECEITA TOTAL, RECEITA EXCLUIDA A RENDA INDUSTRIAL E DESPESAS COM OS SERVICOS DE EDUCAÇÃ, NOS ESTADOS E NO DISTRITO FEDERAL, SEGUNDO OS ORÇAMENTOS DE 1943

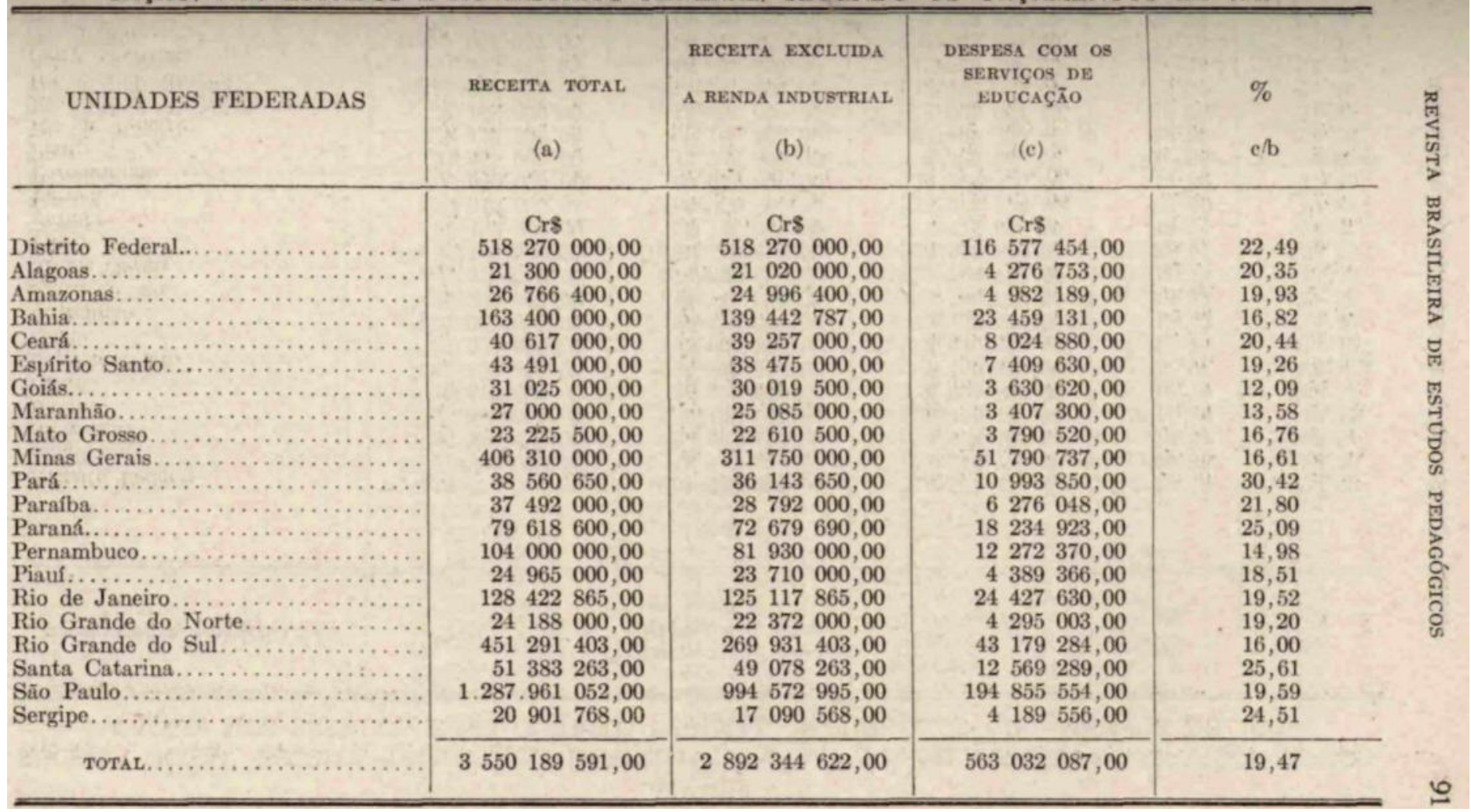


QUADRO II

RECEITA TOTAL, RECEITA TRIBUTARIA (IMPOSTOS E TAXAS) E DESPESAS COM OS SERVIÇOS DE EDUCAÇAO, NOS ESTADOS E NO DISTRITO FEDERAL, SEGUNDO OS ORÇAMENTOS DE 1943

\begin{tabular}{|c|c|c|c|c|c|}
\hline UNIDADES FEDERADAS & $\begin{array}{c}\text { RECEITA TOTAL } \\
(a\end{array}$ & $\begin{array}{c}\text { RENDA TRIBUTÁRIA } \\
\text { (Impostos e taxas } \\
(b)\end{array}$ & $\begin{array}{c}\text { DESPESAS COM OS } \\
\text { SERVIÇOS DE } \\
\text { EDUCAÇÃo } \\
(c)\end{array}$ & $\begin{array}{l}\% \\
c / a\end{array}$ & $\begin{array}{l}\% \\
c / b\end{array}$ \\
\hline+ & Cr\$ & Cr\$ & Cr\$ & & \\
\hline Distrito Federal.............. & 518270000,00 & 401580000,00 & 116577454,00 & 22,49 & 29,03 \\
\hline 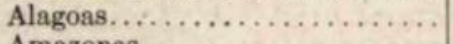 & 21300000,00 & 18135000,00 & 4276753,00 & 20,08 & 23,58 \\
\hline 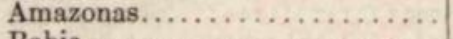 & 26766400,00 & 23684400,00 & $4982 \quad 189,00$ & 18,61 & 21,04 \\
\hline 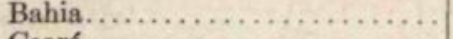 & 163400000,00 & 120197200,00 & 23459131,00 & 14,36 & 19,52 \\
\hline 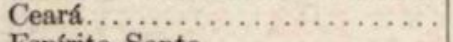 & 40617000,00 & 35922000,00 & 8024880,00 & 19,76 & 22,34 \\
\hline Espirito Santo.............. & 43491000,00 & 29510000,00 & 7409630,00 & 17,04 & 25,11 \\
\hline Goiás...................... & 31025000,00 & 25098000,00 & 3630620,00 & 11,70 & 14,47 \\
\hline 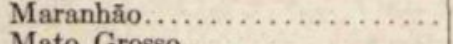 & 27000000,00 & 22870000,00 & 3407300,00 & 12,62 & 14,90 \\
\hline Mato Grosso ................ & 23225500,00 & 17740000,00 & 3790520,00 & 16,32 & 21,37 \\
\hline Minas Gerais................ & 406310000,00 & 274600000,00 & 51790737,00 & 12,75 & 18,86 \\
\hline Pará........................ & 38560650,00 & 28489000,00 & 10993850,00 & 28,51 & 38,59 \\
\hline Paraíba................... & 37492000,00 & 26120000,00 & 6276048,00 & 16,74 & 24,03 \\
\hline 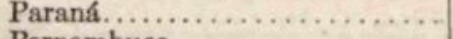 & 79618600,00 & 66050000,00 & 18234923,00 & 22,90 & 27,61 \\
\hline Pernambuco ................. & 104000000,00 & 69920000,00 & 12272370,00 & 11,80 & 17,55 \\
\hline Piau........................ & 24965000,00 & 19213000,00 & 4389366,00 & 17,58 & 22,85 \\
\hline Rio de Janeiro................. & 128422865,00 & 109164500,00 & 24427630,00 & 19,02 & 22,38 \\
\hline Rio Grande do Norte......... . & 24188000,00 & 19551000,00 & 4295003,00 & 17,76 & 21,97 \\
\hline Kio Grande do Sul........... & 451291403,00 & 215237500,00 & $43 \quad 179284,00$ & 9,57 & 20,06 \\
\hline 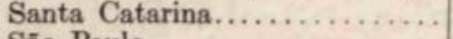 & 51383263,00 & 45823960,00 & 12569289,00 & 24,46 & 27,43 \\
\hline São Paulo................... & 1287961052,00 & 778041865,00 & 194855554,00 & 15,13 & 23,04 \\
\hline Sergipe..................... & 20901768,00 & 16190000,00 & 4189556,00 & 20,04 & 25,88 \\
\hline TotaL............ & $\begin{array}{llll}3 & 550 & 189 & 591,00\end{array}$ & 2363137425,00 & 563032087,00 & 15,86 & 23,83 \\
\hline
\end{tabular}


QUADRO III

RECEITA TRIBUTARIA DE IMPOSTOS, DESPESAS GERAIS COM OS SERVICOOS DE EDUCACAO E COM O ENSINO PRIMARIO, NOS ESTADOS E NO DISTRITO FEDERAL, SEGUNDO OS ORCAMENTOS DE 1943.

\begin{tabular}{|c|c|c|c|c|c|}
\hline UNIDADES FEDERADAS & $\begin{array}{l}\text { RECEITA TRIBUTÁRIA } \\
\text { DE IMPOSTOS } \\
(a\end{array}$ & $\begin{array}{c}\text { DESPESAS COM OS } \\
\text { SERVIÇOS DE } \\
\text { EDUCAÇ̃̃o } \\
(b\end{array}$ & $\%$ & $\begin{array}{c}\text { DESPESAS COM O } \\
\text { ENSINO PRIMÁRIO } \\
(c)\end{array}$ & $\begin{array}{l}\% \\
c / 2\end{array}$ \\
\hline & Cr\$ & $\mathrm{Cr} \$$ & & Cr\$ & \\
\hline Distrito Federal. ............ & 334340000,00 & 116577454,00 & 34,87 & 59165503,00 & 17,70 \\
\hline 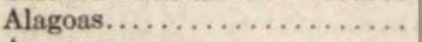 & 17610000,00 & 4286753,00 & 24,29 & 2464620,00 & 14,00 \\
\hline 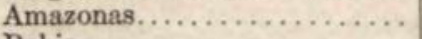 & 16454400,00 & $4982 \quad 189,00$ & 30,28 & 2085650,00 & 12,68 \\
\hline 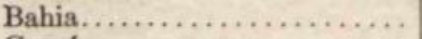 & 105600000,00 & 23459131,00 & 22,22 & 12655768,00 & 11,98 \\
\hline 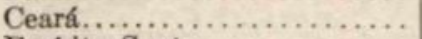 & 35670900,00 & 8024880,00 & 22,50 & 4902221,00 & 13,74 \\
\hline Espírito Santo............... & 26820000,00 & 7409630,00 & 27,63 & 4598560,00 & 17,15 \\
\hline 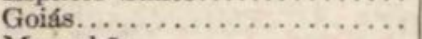 & 23350000,00 & 3630620,00 & 15,35 & 1738872,00 & 7,45 \\
\hline Maranhão.................. & 21575000,00 & 3407300,00 & 15,79 & 2033420,00 & 9,42 \\
\hline Mato Grosso.................. & 15210000,00 & 3790520,00 & 24,92 & 2676920,00 & 17,60 \\
\hline Minas Gerais.............. & 260000000,00 & $59790 \quad 737,00$ & 23,00 & 32799128,00 & 12,61 \\
\hline 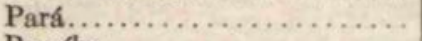 & 26080000,00 & 10993850,00 & 42,25 & 6430200,00 & 24,71 \\
\hline 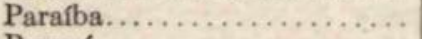 & 25250000,00 & 6276048,00 & 24,86 & $4310 \quad 140,00$ & 17,07 \\
\hline 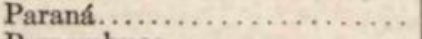 & 56610000,00 & 18234923,00 & 32,21 & 11496061,00 & 20,31 \\
\hline Pernambuco ................ & 65800000,00 & 12272370,00 & 18,65 & 6156320,00 & 9,36 \\
\hline Piaui...$\ldots \ldots \ldots \ldots \ldots \ldots$ & 18420000,00 & 4389366,00 & 23,83 & 2548144,00 & 13,83 \\
\hline Rio de Janeiro ............. & 102854500,00 & 24427630,00 & 23,75 & 16488990,00 & 16,03 \\
\hline Rio Grande do Norte........ & 19175000,00 & 4295003,00 & 22,40 & 2398520,00 & 12,51 \\
\hline Rio Grande do Sul.......... & 206937500,00 & $43 \quad 179284,00$ & 20,87 & 23855200,00 & 11,53 \\
\hline 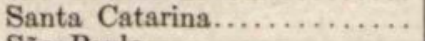 & 43350000,00 & 12569289,00 & 28,99 & 9387976,00 & 21,66 \\
\hline São Paulo................... & 731650000,00 & 194855554,00 & 26,63 & 98898650,00 & 13,52 \\
\hline Sergipe................... & 14020000,00 & 4189556,00 & 29,88 & 1918053,00 & 13,68 \\
\hline TotaL......... & 2166717300,00 & 563032087,00 & 25,99 & 309008886,00 & 14,26 \\
\hline
\end{tabular}




\begin{tabular}{|c|c|c|c|}
\hline$\dot{\ddot{n}}$ & $b^{\circ}$ & 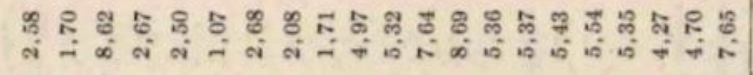 & \\
\hline 以 & 营 & 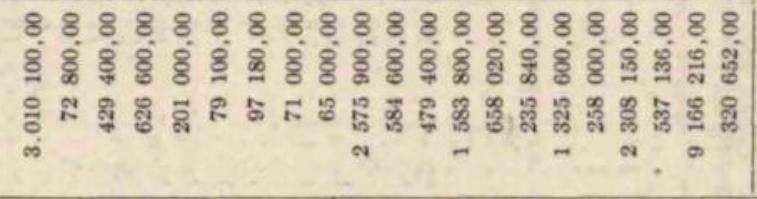 & $\begin{array}{l}8 \\
\text { की } \\
\text { के } \\
\text { ने }\end{array}$ \\
\hline 话是 & $6^{\circ}$ & 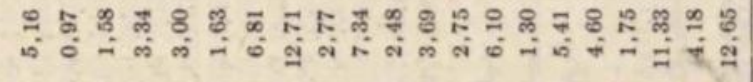 & $\stackrel{\mathscr{6}}{\mathscr{6}}$ \\
\hline 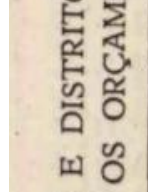 & 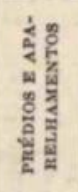 & 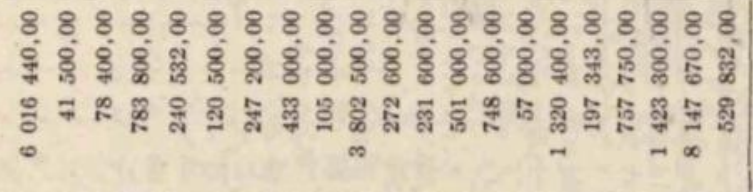 & 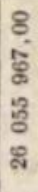 \\
\hline ஜㅇำ & $\Delta$ & 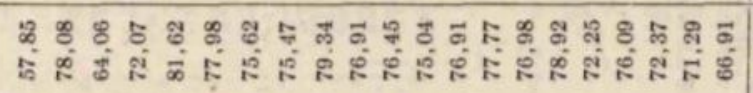 & $\begin{array}{l}\text { है } \\
\text { हृ }\end{array}$ \\
\hline 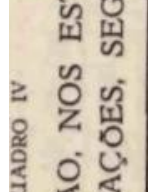 & 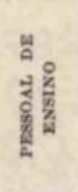 & 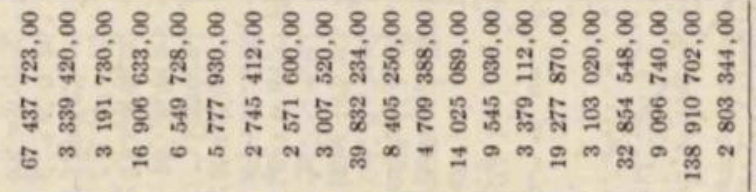 & 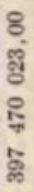 \\
\hline త్రై & $b^{\circ}$ & 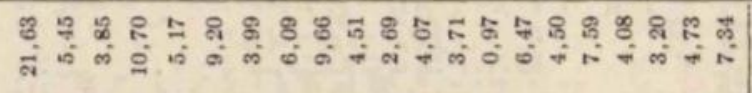 & $\underset{\infty}{\infty}$ \\
\hline 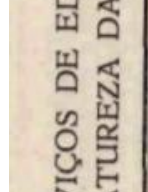 & 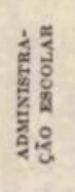 & 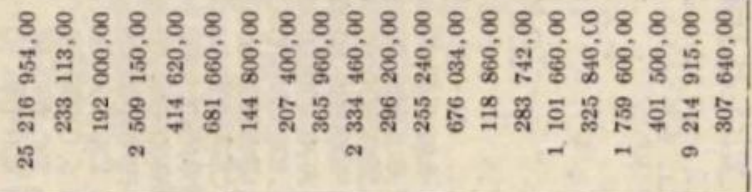 & $\begin{array}{l}8 \\
\text { क्ष } \\
\text { के } \\
\text { Fे }\end{array}$ \\
\hline 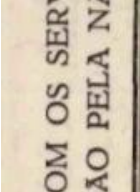 & ta & 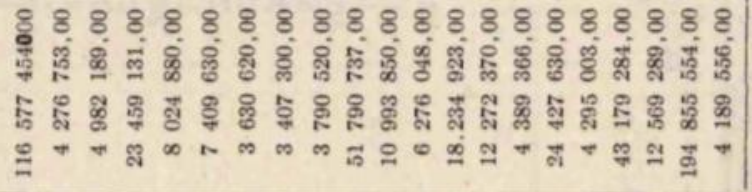 & $\begin{array}{l}\text { : } \\
\text { वू. } \\
\text { of } \\
\text { : }\end{array}$ \\
\hline 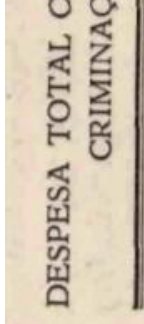 & 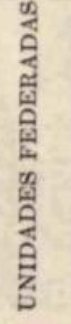 & 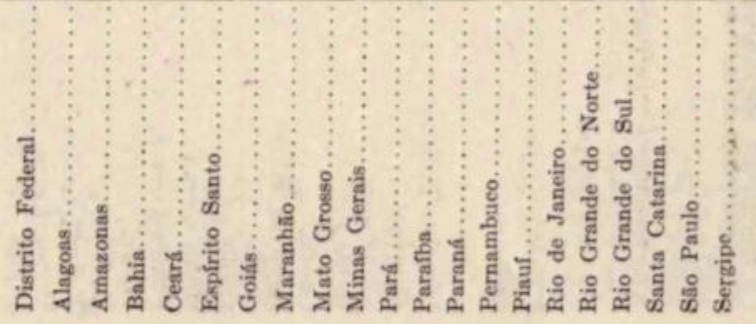 & \\
\hline
\end{tabular}


QUADRO IV (Cont.)

DESPESA TOTAL COM OS SERVIÇOS DE EDUCAÇAO, NOS ESTADOS E DISTRITO FEDERAL, E SUA DISCRIMINAÇAO PELA NATUREZA DAS DOTAÇOES, SEGUNDO OS ORÇAMENTOS DE 1943.

\begin{tabular}{|c|c|c|c|c|c|c|c|c|c|}
\hline UNIDADES FEDERADAS & & $\begin{array}{l}\text { Assist\&XCYA } \\
\text { AO ESCOLAR }\end{array}$ & $\%$ & stvex cõ̃s & $\%$ & $\begin{array}{l}\text { DIFUsĩo } \\
\text { CULTURAL }\end{array}$ & $\%$ & $\begin{array}{c}\text { oOTrAs } \\
\text { DEsPEsAs }\end{array}$ & $\%$ \\
\hline Distrito Federal. ......................... & 4 & 176100,00 & 3,58 & - & 一 & $10462 \quad 137,00$ & 8,98 & 258000,00 & 0,22 \\
\hline 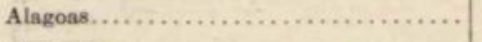 & & - & 一 & 460000,00 & 10,76 & 37720,00 & 0,88 & 92200,00 & 2,16 \\
\hline 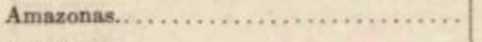 & & 188400,00 & 3,78 & 110200,00 & 2,21 & 612059,00 & 12,29 & 180000,00 & 3,61 \\
\hline 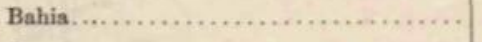 & & 612110,00 & 2.61 & 274800,00 & 1,17 & 362139,00 & 1,54 & 1383899,00 & 5,90 \\
\hline 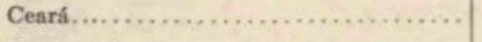 & & 33000,00 & 0,41 & 220000,00 & 2,74 & 56700,00 & 0,71 & 309300,00 & 3,85 \\
\hline Espírito Santo .............................. & & 163200,00 & 2,20 & 170000,00 & 2,29 & 91760,00 & 1,24 & 325480,00 & 4,39 \\
\hline Goiás..................... & & - & - & 180400,00 & 4,97 & 200528,00 & 5,52 & 15100,00 & 0,41 \\
\hline 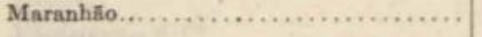 & & 30000.00 & 0,88 & 一 & - & 43500,00 & 1,28 & 50800,00 & 1,49 \\
\hline 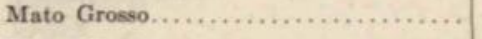 & & - & - & 156800,00 & 4,14 & 68240,00 & 1,80 & 22000,00 & 0,58 \\
\hline Minas Gerais................. & & 832580,00 & 3,54 & - & - & 128400,00 & 0,25 & 1284663,00 & 2,48 \\
\hline 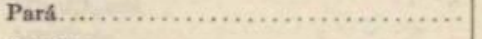 & & 216000,00 & 1,97 & 563800,00 & 5,13 & 422200,00 & 3,84 & 233200,00 & 2,12 \\
\hline Parafba................ & & 255080,00 & 4,06 & 50000,00 & 0,80 & 238700,00 & 3,80 & 56640,00 & 0,90 \\
\hline Parand...................... & & 9720,00 & 0,05 & 743000,00 & 4,07 & 85000,00 & 0,47 & 611300,00 & 3,35 \\
\hline Pernambuco................................. & & 277000,00 & 2,26 & 571960,00 & 4.66 & 191300,00 & 1,56 & 161600,00 & 1,32 \\
\hline Piauf................ & & 一 & - & 330320,00 & 7,53 & 88352,00 & 2,01 & 15000,00 & 0,34 \\
\hline Rio de Janeiro......... & & 200000,00 & 0,82 & 270000,00 & 1,11 & 814500,00 & 3,33 & 117600,00 & 0,48 \\
\hline Rio Grande do Norte............... & & 21600,00 & 0,50 & 296400,00 & 6,89 & 12800,00 & 0,30 & 100000,00 & 2,33 \\
\hline Rio Grande do Sul... & & 441000,00 & 1,02 & 4091000,00 & 9,47 & 857375,00 & 1,99 & 109861,00 & 0.25 \\
\hline Santa Catarina .................... & & 509550,00 & 4,05 & 449860,00 & 3,58 & 133460,00 & 1,06 & 17743,00 & 0,14 \\
\hline 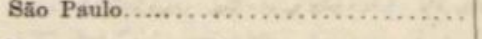 & & 658540,00 & 2,90 & 3576500,00 & 1,84 & 4377529,00 & 2,25 & 15803482,00 & 8,11 \\
\hline \multirow[t]{2}{*}{ 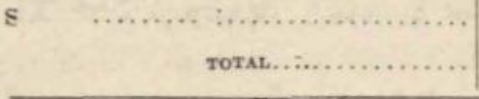 } & & 38900,00 & 0,93 & 88700,00 & 2,12 & 87288,00 & 2,08 & 13200,00 & 0,32 \\
\hline & & 662780,00 & 2,60 & 12603740,00 & 2,24 & 19371687,00 & 3,44 & 21161068,00 & 3,76 \\
\hline
\end{tabular}


DESPESA TOTAL, COM O ENSINO PROPRIAMENTE DITO, E SUA DISCRIMINAÇAO PELOS VARIOS RAMOS E GRAUS DE ENSINO, NOS ESTADOS E NO DISTRITO FEDERAL, SEGUNDO OS ORÇAMENTOS DE 1943

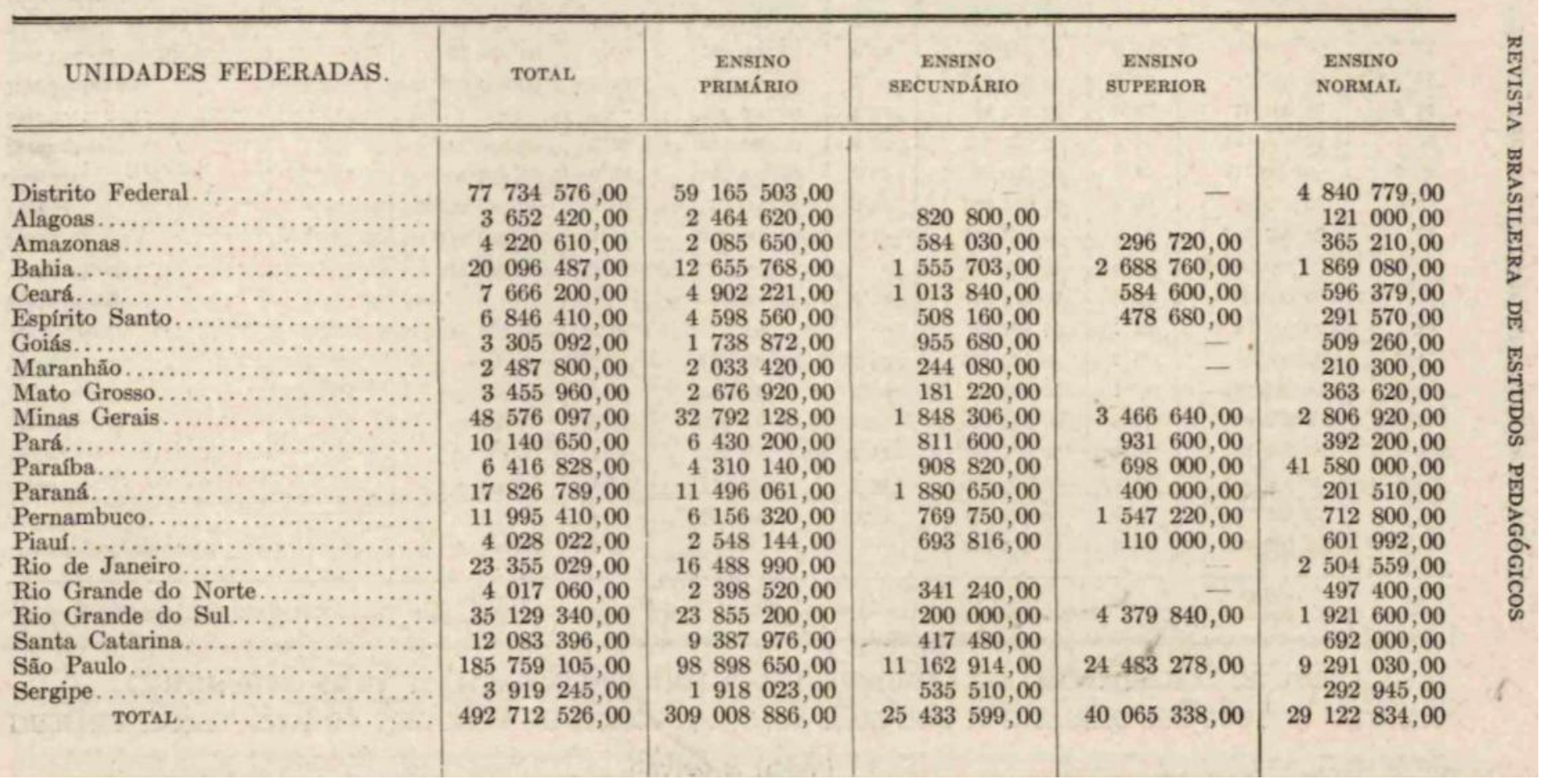


QUADRO v (Cont.)

DESPESA COM O ENSINO PROPRIAMENTE DITO E SUA DISCRIMINAÇAO PELOS VARIOS RAMOS

E GRAUS DE ENSINO, NOS ESTADOS E DISTRITO FEDERAL, SEGUNDO OS ORÇAMENTOS DE 1943.

\begin{tabular}{|c|c|c|c|c|c|c|}
\hline UNIDADES FEDERADAS & $\begin{array}{c}\text { ENsISO } \\
\text { INDCRTRLAL } \\
\text { E PROTSSIONAL } \\
\text { EM GERAL }\end{array}$ & $\begin{array}{c}\text { ENEINO } \\
\text { AGRfCoL.A }\end{array}$ & $\begin{array}{c}\text { ENSINO } \\
\text { EMEXDATIVO }\end{array}$ & $\begin{array}{c}\text { ENsixo } \\
\text { DE ADULTOS }\end{array}$ & $\begin{array}{c}\text { ZNSINo } \\
\text { COSIERCIAL }\end{array}$ & DTVERSOS \\
\hline Distrito Federal........ & $5614 \quad 575,00$ & - & 1165000,00 & - & - & 6948719,00 \\
\hline Alagoas.................. & 195300,00 & - & - & - & - & 50700,00 \\
\hline Amazonas ................. & - & - & 772800,00 & 6000,00 & - & $110 \quad 200,00$ \\
\hline Bahia......................... & 97760,00 & - & 946856,00 & -1 & - & 282560,00 \\
\hline Ceara ............. & - & 58200,00 & 198520,00 & 20440,00 & - & 292000,00 \\
\hline Espírito Santo & 33360,00 & 490600,00 & 170520,00 & 12000,00 & - & 262960,00 \\
\hline Goisen........................ & 36000,00 & - & 36500,00 & 20380,00 & - & 8400,00 \\
\hline Maranhđ̃o............... & - & - & - & - & - & - \\
\hline Mato Grosso............ & - & - & 24000,00 & - & 191200,00 & 19000,00 \\
\hline Minas Gerais ..... & - & 2740380,00 & 1406523,00 & 216000,00 & 8500,00 & 3283700,00 \\
\hline Pará..................... & 969200,00 & - & 7200,00 & 33000,00 & - & 565650,00 \\
\hline Parafba .................. & 163560,00 & - & 220728,00 & - & - & 74000,00 \\
\hline Paraní, .................... & 51708,00 & 2374620,00 & 200600,00 & - & - - & 1221640,00 \\
\hline Pernambuco ............ & 768960,00 & 62800,00 & 132600,00 & - & - & 1844960,00 \\
\hline Piamf ............... & - & 5000,00 & - & 3720,00 & 10800,00 & 54550,00 \\
\hline Rio de Janeiro........ & 2576860.00 & 287100,00 & 610760,00 & 80000.00 & - & 876760,00 \\
\hline Rio Grande do Norte.... & 102240,00 & - & 147060,00 & 4200,00 & - & 526400,00 \\
\hline Rio Grande do Sul........ & 600000,00 & $2014=-$ & 755700,00 & 18000,00 & 298000,00 & 3101000,00 \\
\hline Santa Catarina .......... & 59250,00 & 114720,00 & 713550,00 & 4800,00 & - & 693620,00 \\
\hline Sáo Paulo..................... & $9.890 \quad 100,00$ & $17710 \quad 173,00$ & 8061630,00 & 1462150,00 & $x=-$ & $4799 \quad 180,00$ \\
\hline Sergipe.............. & $466 \quad 450,00$ & - & $257+477,00$ & 3200,00 & 181540,00 & 265000,00 \\
\hline TOTAL ........... & 21625323,00 & 23843593,00 & 15828024,00 & 1883890,00 & 690040.00 & 25210999.00 \\
\hline
\end{tabular}


QUADRO vi

DISTRIBUIÇÃO PERCENTUAL DAS DESPESAS COM O "ENSINO PROPRIAMENTE DITO ", SEGUNDO OS VARIOS GRAUS E RAMOS DO ENSINO, NOS ESTADOS E DISTRITO FEDERAL, EM 1943

\begin{tabular}{|c|c|c|c|c|c|c|c|c|c|c|}
\hline UNIDADES FEDERADAS & preximo & szeuxpíno & strenton & sonval: & 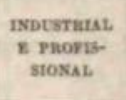 & AeríoLA & EREXOATTVO & ADELTOS & COMERCial & nevensos \\
\hline Distrito Federal.. & 76,11 & - & - & 6,23 & 7,22 & - & 1,50 & - & - & 8,94 \\
\hline Alagoas ............ & 67,48 & 22,47 & - & 3,31 & 5,35 & $=$ & - & - & - & 1,39 \\
\hline Amazonas. & 49,42 & 13,84 & 7.03 & 8,65 & - & - & 18,31 & 0,14 & - & 2,61 \\
\hline Bahia..... & 62,97 & 7,74 & 13,38 & 9,30 & 0,49 & - & 4,71 & - & - & 1,41 \\
\hline Ceará ............... & 63,95 & 13,22 & 7.63 & 7,78 & - & 0,76 & 2,59 & 0,27 & - & 3,80 \\
\hline Espirito Santo. & 67,16 & $-7,42$ & 6.90 & 4,26 & 0.49 & 7,17 & 2,49 & 0,18 & - & 3,84 \\
\hline Goiks............... & 52,61 & 28,92 & - & 15.41 & 1,09 & - & 1,10 & 0,62 & - & 0,25 \\
\hline Maranhăo & 81,74 & 9,81 & - & 8,45 & - & - & $\overline{-}$ & - & $-\bar{c}$ & $=$ \\
\hline Mato Grosso..... & 77,46 & 5,24 & - & 10,52 & - & - & 0.70 & - & 5,53 & 0,55 \\
\hline Minas Gernis. & 67,52 & 3,80 & 7,14 & 5,75 & - & 5,64 & 2,90 & 0,44 & 0.02 & 6.76 \\
\hline Park . ........... & 63,41 & 8,00 & 9,19 & $3.8 \%$ & $9: 50$ & - & 0,07 . & 0.23 & - & 3,37 \\
\hline Parafbe. & 67,17 & 14,16 & 10,88 & 0,65 & 2,55 & - & 3,44 & - & - & 1,15 \\
\hline Paraná ....... & 64,49 & 10,55 & 2,24 & 1,13 & 0,29 & 13,32 & 1,13 & - & - & 6,85 \\
\hline Pernatubuco ...... & 51,32 & 6,42 & 12,90 & 5,04 & 6.41 & 0,52 & 1.11 & - & - & 15.38 \\
\hline Piaui. ............. & 63,26 & 17,23 & 2,73 & 14,95 & - & 0,12 & - & 0,09 & $-0,27$ & 1,35 \\
\hline 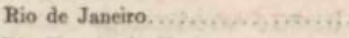 & 70,60 & - & - & 10,72 & $-11,03$ & 1,23 & 2,62 & 0,34 & - & 3,46 \\
\hline Rio Grande do Norte................. & 59,71 & 8,50 & - & 12,38 & 2,55 & - & 3,66 & 0,10 & - & 13.10 \\
\hline Rio Grande do Sul ....... & 67,91 & 0,57 & 12,47 & 5,47 & 1,71 & - & 2,15 & 0,05 & 0.85 & 8.82 \\
\hline Santa Catarina ..... & 77,69 & 3,45 & - & 5,73 & 0,49 & 0,95 & 5,91 & 0.04 & - & 5,74 \\
\hline São Paulo ........ & 53.24 & 6,01 & 13,18 & 5,00 & 5,33 & 9,53 & 4,34 & 0,79 & - & 2,58 \\
\hline \multirow[t]{2}{*}{ Serzipe..... } & 48,94 & 13,67 & - & 7,45 & 11,90 & - & . 6,57 & 0,05 & 4.63 & 6.76 \\
\hline & 62,72 & 5,16 & 8,13 & 5,91 & 4,39 & 4,84 & 3,21 & 0,38 & 0,14 & 5,12 \\
\hline
\end{tabular}


DESPESA "PER CAPITA" EM RELACAO A DESPESA TOTAL E A DESPESA COM OS SERVICOS DE EDUCA. ÇÃO, NOS ESTADOS E NO DISTRITO FEDERAL, SEGUNDO OS ORÇAMENTOS DE 1943.

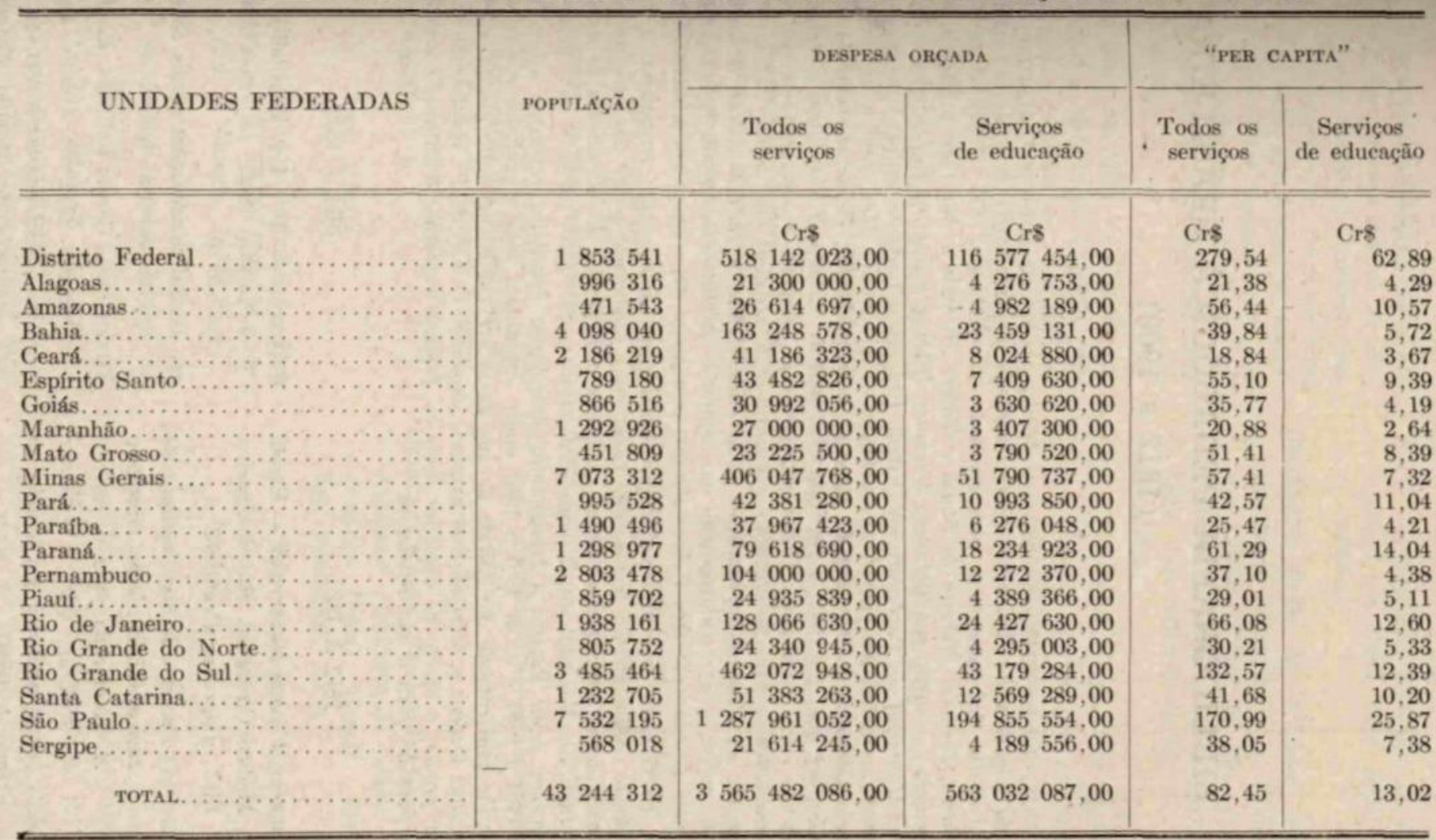

Seçĩo de Inquéritos e Pesquisas do I.N.E.P. - Visto (a) Manoel Marques de Carvatho, chefe de scgâo. 